\title{
The Dlgitizer ReAdout Controller (DIRAC) of the Mu2e electromagnetic calorimeter at Fermilab
}

\author{
Elena Pedreschi ${ }^{1}$, Franco Cervelli, Stefano Di Falco, Simone Donati, Luca \\ Morescalchi, Fabrizio Raffaelli, Franco Spinella \\ INFN Sezione di Pisa \\ Ed. C Polo Fibonacci, Largo Pontecorvo 3, Pisa, Italy \\ E-mail: elena.pedreschi@pi.infn.it
}

Sergio Ceravolo, Gianni Corradi, Eleonora Diociaiuti, Raffaella Donghia, Simona Giovannella, Fabio Happacher, Matteo Martini, Stefano Miscetti, Daniele

Pasciuto, Ivano Sarra

Laboratori Nazionali di Frascati dell'INFN

Via E. Fermi 40, 00044 Frascati, Italy

\begin{abstract}
We report on the design and performance of the Digitizer ReAdout Controller (DIRAC) of the Mu2e electromagnetic calorimeter, which consists of a 670 CsI crystals matrix readout by SiPM. The 20-channels DIRAC performs $200 \mathrm{MHz}$ sampling of the SiPM signals transmitted by the front-end electronics. Operation in the Mu2e hostile environment expected Total Ionizing Dose (TID) of $12 \mathrm{Krad}$ and neutron fluence of $5 \times 10^{10} \mathrm{n} / \mathrm{cm}^{2} @ 1 \mathrm{MeV}_{\text {eq }}(\mathrm{Si}) / \mathrm{y}, 1 \mathrm{~T}$ magnetic field, level of vacuum of $10^{-4}$ Torr has made the DIRAC design challenging. We describe design, specifications, architecture and results of the tests performed on the DIRAC prototypes.
\end{abstract}

TWEPP 2019 Topical Workshop on Electronics for Particle Physics

2-6 September 2019

Santiago de Compostela, Spain

${ }^{1}$ Speaker 


\section{Introduction}

The Mu2e experiment [1] will search for Charged Lepton Flavour Violation looking at the conversion of a muon into an electron in the field of an aluminium nucleus. The Standard Model of particle physics, even extendend to include the finite neutrino masses, predicts the ratio $R \mu e$ between muon conversions and muon nuclear captures to be $10^{-52}$. Several extensions of the Standard Model predict $R \mu$ e to be in the range of $10^{-14} \div 10^{-18}$. The Mu2e experiment plans to improve the experimental limit, set actually by the SINDRUM II experiment, by four order of magnitude to test many of the possible extensions of the Standard Model .

To reach this ambitious goal, the Mu2e experiment will use an intense pulsed muon beam, and rely on a detector system composed of a straw tube tracker and a calorimeter made of pure CsI crystals.

The calorimeter plays a central role in the Mu2e measurement, providing particle identification and the calorimeter information allows the improvement of the tracking performance. Thanks to a calorimeter-seeded track finder algorithm, it is possible to increase the track reconstruction efficiency, and make it more robust with respect to the occupancy level.

\subsection{Mu2e experiment layout}

In the Mu2e detector [2] a graded magnetic field inside a Production Solenoid (from 4.6T to $2.5 \mathrm{~T}$ ), reflects slow forward $\mu / \pi$ and contains backward $\mu / \pi$ produced by a $8 \mathrm{GeV}$ proton beam that hits a tungsten target. Inside the Transport Solenoid the $\pi$ decay to $\mu$, the low momentum $\mu^{-}$ are selected and transported towards the Detector Solenoid (DS) where they are stopped on Al foils and trapped in orbit around the nucleus. Inside the Detector Solenoid a strobe-tube tracker, an electromagnetic calorimeter and an external veto for cosmic rays surrounding the solenoid optimized for $105 \mathrm{MeV}$ momentum reconstruct events (Figure 1).

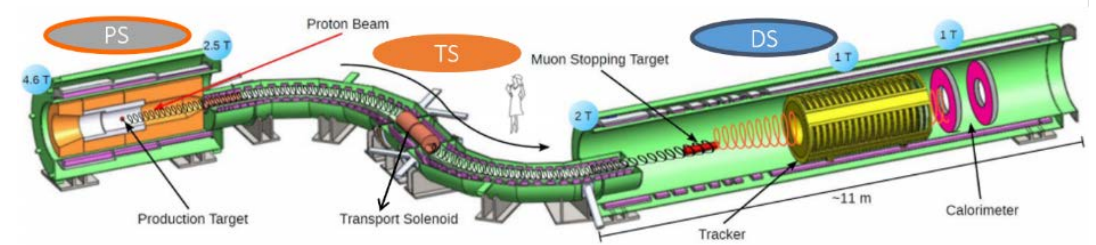

Figure 1. Mu2e detector overview. The production target is inside the Production Solenoid, while the Stopping target and the detectors are located inside the Detector Solenoid.

\subsection{The electromagnetic calorimeter}

The Mu2e calorimeter plays an important role in providing particle identification, which consists of e/ $\mu$ separation rejecting $\mu$ background, improve the track pattern recognition and a standalone trigger.

The calorimeter [3] is a high granularity crystal calorimeter consisting of 1348 un-doped CsI crystals, arranged in two disks. Each crystal is coupled to two large-area UV-extended SiPMs arrays. Each array consists of two series of three SiPMs. The two series are connected in parallel by the Front End electronics to have a double redundancy. The calorimeter is located in a very harsh environment with a $10^{-4}$ Torr vacuum, a uniform magnetic field of $1 \mathrm{~T}$ and exposed to a very intense particle flux (TID $90 \mathrm{krad} / 5$ year for crystals and $75 \mathrm{krad} / 5$ year for sensors, NIEL 3x10 12 $\mathrm{n} / \mathrm{cm}^{2}$ for crystals and $1.2 \times 10^{12} \mathrm{n} / \mathrm{cm}^{2}$ for sensors). 
The Mu2e calorimeter requirements to detect $105 \mathrm{MeV}$ electrons are a energy resolution $\sigma_{\mathrm{E}} / \mathrm{E}$ $<10 \%$, timing resolution $\sigma(\mathrm{t})<500 \mathrm{ps}$, position resolution $<1 \mathrm{~cm}$.

\section{The calorimeter readout electronics}

The magnet cryostat hosts all the calorimeter Data AcQuisition (DAQ) system in order to limit the number of pass-through connectors. The DAQ system is disposed around the external radius of the calorimeter disks. On the other hand, this solution complicates the design in terms of available space and accessibility, in case of failure, and power dissipation.

In addition, the digitizer boards have to stand all the radiation coming directly from the interaction in the stopping target, mainly bremsstrahlung photons from the beam flash and neutrons from muon captures. Most of the charged particles are trapped at the lowers radius by the solenoidal field and cannot reach the crates. GEANT4 and MARS simulation shows that in three years of data taking, the digitizer components will absorb a total ionizing dose of $12 \mathrm{krad}$ (safety factor included) and will suffer displacement damage equivalent to the one produced by a flux of $5 \times 10^{10} \mathrm{n} / \mathrm{cm}^{2} @ 1 \mathrm{MeV}_{\text {eq }}(\mathrm{Si}) / \mathrm{y}$. The simultaneous presence of radiation and high magnetic field imposes stringent constraints on the component choice.

To satisfy the requirements of the calorimeter, in terms of time and energy resolution, Montecarlo simulations were made. Table 1 shows simulation results, in terms of time and energy resolution. The table shows that a digitizer with sampling frequency of $200 \mathrm{MHz}$ and an ADC with 12 bit resolution matches the calorimeter requirements.

In terms of electrical signal, the Signal to Noise Ratio (SNR) must be less than the LSB, with the analog to digital converter used in the DIRAC, which has dynamics $\pm 1 \mathrm{~V}$, the SNR is 0.5 $\mathrm{mV}$.

\begin{tabular}{|c|c|c|c|}
\hline & $150 \mathrm{MHz}$ & $200 \mathrm{MHz}$ & $250 \mathrm{MHz}$ \\
\hline 8 bits & $470 \mathrm{ps} / 9.8 \mathrm{MeV}$ & $440 \mathrm{ps} / 8.0 \mathrm{MeV}$ & $440 \mathrm{ps} / 7.8 \mathrm{MeV}$ \\
\hline 10 bits & $370 \mathrm{ps} / 6.5 \mathrm{MeV}$ & $250 \mathrm{ps} / 5.5 \mathrm{MeV}$ & $250 \mathrm{ps} / 5.5 \mathrm{MeV}$ \\
\hline 12 bits & $300 \mathrm{ps} / 6.2 \mathrm{Mev}$ & $\mathbf{1 7 0} \mathbf{p s} / \mathbf{5 . 5} \mathbf{M e V}$ & $170 \mathrm{ps} / 5.5 \mathrm{MeV}$ \\
\hline
\end{tabular}

Tabelle 1. Time / Energy resolution vesus sampling frequency and ADC-bits

\subsection{The Front End Electronics and the Mezzanine Board}

The Front End Electronics (FEE) consists of two discrete and independent chips (Amp-HV), for each crystal, directly connected to the back of the SiPM pins. These provide the amplification and shaping stage, a local linear regulation of the bias voltage, monitoring of current and temperature on the sensors and a test pulse. A dedicated mezzanine board (MB) equipped with an ARM processor controls a groups of 20 Amp-HV chips, distribute the low voltage and the high voltage reference values, set and read back the locally regulated voltages. Groups of 20 differential signals are sent to the waveform digitizer board called DIRAC.

\subsection{DIRAC architecture}

The core of the DIRAC board is a large FPGA SoC (MicroSemi ${ }^{\circledR}$ SmartFusion2 M2S150T), that handles 10 ultralow-power double channels 12 bits and maximum sample rate of 250 MSPS analog-to-digital converters ADCs (Texas Instruments ${ }^{\circledR}$ ADS4229) protocol and timing, sparsifies and compresses the digitized data and forms a packet that is sent optically, thru a dual fiber optical transceiver (Cotswork® RJ-5G-SX-DPLX) to the event builder using a custom protocol. 
A ultra-low noise clock jitter cleaner (Texas Instruments ${ }^{\circledR}$ LMK04828) provides a high performance clocking tree in the board. The power on the board is handled by 4 DC-DC converters (Linear Technologies ${ }^{\circledR}$ LTM8033) and combined with 6 LDO (Micrel ${ }^{\circledR}$ MIC69502)

The DIRAC board is $233 \mathrm{~mm} \times 165 \mathrm{~mm}$, has 16 layers realized in FR408-HR with a thickness of $2.127 \mathrm{~mm}, 100 \Omega$ differential lines and $50 \Omega$ single ended lines.

\section{Qualification tests}

The harsh operating environment requires testing and qualifying all the components. Damage inflicted to the electronic devices by a single particle (Single Event Effect - SEE) can be temporary or permanent. The Total Ionizing Dose (TID) effect, for which the accumulated influence causes degradation, is treated separately from the displacement damage induced by the Non-Ionizing Energy-Loss (NIEL) deposition.

Temporary effects like bit flipping (Single Event Upset) are more related to digital or mixed logic and for this version of the DIRAC are not implemented. The adopted FPGA is already qualified by the producer as SEL free and for a very low SEU probability. Several test campaigns has been performed for all the others components.

TID up to 20 krad tests were performed in gELBE@HZDR $\gamma$ from Bremsstrahlung $(0<\mathrm{E}<14 \mathrm{MeV})$ and in Calliope @ENEA facility with a Co60 source.

Neutrons tests has been performed in gELBE @HZDR $\left(1.06 \times 10^{12} \mathrm{n} / \mathrm{cm}^{2}\right)$ and in ENEA $\left(6 \times 10^{11} \mathrm{n} / \mathrm{cm}^{2}\right)$.

Magnetic Field tests $(\mathrm{B}=1 \mathrm{~T})$ for DC-DC converters has been done in LASA@INFN Milano.

In particular the ADC has been tested up to $20 \mathrm{krad}$ and $6 \times 10^{12} 1 \mathrm{MeV}(\mathrm{Si}) \mathrm{n} / \mathrm{cm}^{2}$ digitizing a $200 \mathrm{kHz}$ sinusoidal signal and converting it back to the analog signal. We have analyzed more than 300 GB of data from both tests and we have observed no evidence waveform shape variation [4]. The DCDC converter has been tested up to $20 \mathrm{krad}$, observing an increase of the output voltage of about $0.5 \mathrm{~V}$ at $20 \mathrm{krad}$ (limit is $12 \mathrm{krad}$ ). An irradiation of $0.5 \times 10^{12} 1 \mathrm{MeV}$ (Si) $\mathrm{n} / \mathrm{cm}^{2}$ cause an increase of $40 \mathrm{mV}$.

The DC-DC converter test result in a B = 1T shows that the currents tends to increase slightly with the increase of the applied magnetic field, with a consequent decrease in conversion efficiency. The device provides still the same output voltage.

\section{Slice test}

A large scale EMC prototype, named Module0, was realized: 51 CsI crystals, $102 \mathrm{Mu2e}$ SiPMs, 102 FEE boards with mechanics and cooling system similar to the final ones.

The Module 0 goals are multiple: validate the acquisition chain (Slice Test), evaluate the performances of acquisition chain with electron beam @ $60 \div 120 \mathrm{MeV}$, test integration and assembly procedures and irradiation tests.

To validate analog part of the DIRAC in terms of dynamic, SNR, analog bandwidth and linearity a full chain test, with Cosmic ray, 1 CsI Crystal (final), FEE board, Mezzanine board and DIRAC, was performed. To validate the system a vertical slice test connecting for the first time the Module 0 equipped with FEE and the SiPM cooled at $18{ }^{\circ} \mathrm{C}$ degrees with the DIRAC board and the Mezzanine board to 16 channels of the Module 0 .

The test demonstrated that the DIRAC analog section works fine: the signal shape are as expected from simulation and the time resolution (0.50/SQRT(2)) is $350 \mathrm{ps}$ (Figure 2). 


\section{Conclusion}

The working environment of Mu2e calorimeter sets several constrains in the DIRAC design. At the present, the first version of the Mu2e waveform digitizer has been defined, designed and tested for TID and NIEL. Power, analog and sampling sections, have been tested and qualified to operate in high magnetic field and to survive a TID of $20 \mathrm{krad}$ and a neutron flux up to $1.06 \times 10^{12} \mathrm{n} / \mathrm{cm}^{2}$.

A second version of DIRAC board is under design with newer DC-DC converter, qualified up to $48 \mathrm{krad}$ and a newer FPGA more powerful respect to SmartFusion2 and with configuration cells Single Events Effects (SEE) immune.
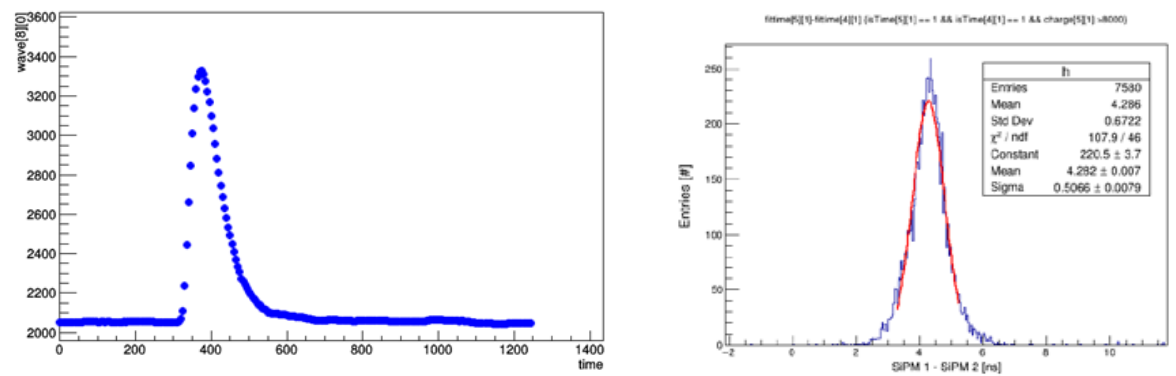

Figure 2. DIRAC slice test example of resulting waveform and time resolution.

\section{Acknowledgments}

We are grateful for the vital contributions of the Fermilab staff and the technical staff of the participating institutions. This work was supported by the US Department of Energy; the Itacomponents lian Istituto Nazionale di Fisica Nucleare; the Science and Technology Facilities Council, UK; the Ministry of Education and Science of the Russian Federation; the US National Science Foundation; the Thousand Talents Plan of China; the Helmholtz Association of Germany; and the EU Horizon 2020 Research and Innovation Program under the Marie Sklodowska-Curie Grant Agreement No. 690385, No. 734303, and No. 822185. Fermilab is operated by Fermi Research Alliance, LLC under Contract No. De-AC02-07CH11359 with the US Department of Energy, Office of Science, Office of High Energy Physics

\section{References}

[1] R.H. Bernstein, The Mu2e Experiment, 2019 Front. Phys. 7:1. doi: 10.3389/fphy.2019.00001

[2] Mu2e collaboration, Mu2e technical Design report, 2015, arXiv:1501.05241.

[3] Mu2e collaboration, Mu2e Calorimeter Final technical Design report, 2018, arXiv: 1501.05241.

[4] S. Di Falco, et al., Components qualification for a possible use in the Mu2e calorimeter waveform digitizer, J. Instrum. 12 (2017). 